\title{
Welcome Message from the Editors-in-Chief
}

\author{
James Joshi ${ }^{1}$, Calton $\mathrm{Pu}^{2}$ \\ ${ }^{1}$ University of Pittsburgh, Pittsburgh, USA \\ ${ }^{2}$ Georgia Institute of Technology, Atlanta, USA
}

Received on 14 May 2014, published on 27 May 2014

Copyright ( 2014 James Joshi and Calton Pu, licensed to ICST. This is an open access article distributed under the terms of the Creative Commons Attribution licence (http://creativecommons.org/licenses/by/3.0/), which permits unlimited use, distribution and reproduction in any medium so long as the original work is properly cited.

doi: 10.4108/cc.1.1.e1

On behalf of the Editorial Board and the Advisory Board, we are pleased to welcome all to the inaugural issue of the EAI Endorsed Transactions on Collaborative Computing. This journal reflects the increasing maturity of the growing community of researchers involved in various areas of collaborative systems, networks, infrastructures and applications. The journal is also aligned with our IEEE/EAI International Conference on Collaborative Computing: Networking, Applications and Worksharing (IEEE/EAI CollaborateCom) which has nurtured the community. We could not find a better timing for this inaugural issue than the $10^{\text {th }}$ anniversary of IEEE/EAI CollaborateCom that will be held in Miami, USA on Oct 22-25, 2014.

Given the ongoing rapid advances in computing and information technologies, we see unprecedented collaboration opportunities for individuals and distributed teams of humans, computer systems and applications, and highly heterogeneous computing devices. The contributions from the community have evolved from standalone tools to open systems, and further, from general purpose tools to specialized collaboration infrastructures that facilitate intensive collaboration in multi-organizational settings, reaching towards global scale social interactions and worksharing. Such collaboration-enhancing technologies enable large and globally dispersed organizations to achieve a much higher level of productivity and efficiency while accelerating innovation.

The goal of this journal is to serve as a premier international venue for publishing innovative and cutting edge research results in theory as well as applied systems, applications and networking areas that enable intensive and efficient collaboration across and among networked cyberphysical and social systems and applications, individuals as well as enterprises. Novel collaboration solutions that fully realize the promises of electronic collaboration, and pushes the limits of human endeavor, productivity and discovery require innovations and advancements in broad areas of computing including networking, systems and applications, user interfaces and interaction paradigms, and seamless interoperation among system, network and application- specific components and tools. Sample topics of interest include, but are not limited to:

- Participatory sensing, crowdsourcing, and citizen science,

- Theoretical foundations and algorithms for collaborative networks, applications, and infrastructures,

- Architectures, protocols, and enabling technologies for collaborative networks, systems and applications

- Autonomic computing and quality of services in collaborative networks, systems, and applications

- Collaboration in context-aware, pervasive, cloud computing, and social networking environments

- Cyber physical and Cyber human environments, and Internet of Things (IoT)

- Collaborative multimedia systems/applications, large scale databases and BigData

- Collaboration in e-health, e-learning, e-government, and digital libraries

- Collaborative network infrastructures such as body area networks, wireless sensor networks, VANETS, mobile networks, etc.

- Models and mechanisms for real-time and large scale collaboration, information and work sharing

- Security, privacy, resiliency/survivability and trust management in collaborative networks, systems, and applications

- Simulation, performance evaluation, experiments, and case studies of collaborative environments

- Collaborative web services technologies, business processes, workflows, and service-oriented systems and architectures

- $\quad$ Etc.

To highlight the core community this journal shares with IEEE/EAI CollaborateCom conference, we have created this inaugural publication as a special issue of extended versions of the five best papers selected from IEEE/EAI Collaborate 2013 which was held in Austin, Texas, USA in October, 2013. We express our immense gratitude to the Guest Editors - Surya Nepal (CSIRO Computational Informatics, 
Australia), Mudhakar Srivatsa (IBM Research, USA), Alessandro Vinciarelli (University of Glasgow, UK), Elisa Bertino, (Purdue University, USA), and Dimitrios Georgakopoulos (CSIRO Computational Informatics, Australia) - for managing the additional reviews and revisions for the journal. We also thank the reviewers for providing timely reviews to meet our deadlines. Please see the Guest Editorial for an overview of the papers.

Complementing the five reviewed papers, we invited a special contribution by the keynote speaker at the conference: Professor Mahadev Satyanarayanan of Carnegie Mellon University (USA), on one of the most exciting and enlightening keynote speech given at IEEE/EAI CollaborateCom. The keynote highlights, and the invited paper details, the explosive growth of executable content and a need to share and archive them at Internet-scale to facilitate spatial and temporal collaboration.

The creation of a journal is the culmination of the achievements of an entire research community. With this inaugural issue, we look forward to an even brighter and grander journey of the community, towards publication and archiving of premier quality, highly innovative and ground breaking research results. We encourage and eagerly expect your paper submissions, proposals for Special Issues, and suggestions towards making this journal a premier quality venue for the community.

Last but not the least, we give special thanks to Imrich Chlamatac, President of European Alliance for Innovation (EAI) for his invaluable support and guidance in creating this journal. We also sincerely thank Sara Funer, Volha Shaparava and the publication staff from EAI for their guidance and support.

\section{About the Editors-in-Chief}

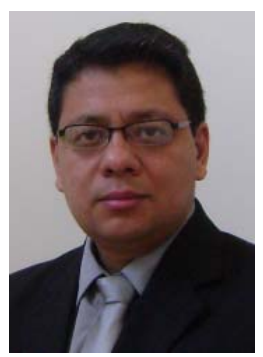

James Joshi (University of Pittsburgh, USA) was born in Nepal. He is an associate professor in the School of Information Sciences at the University of Pittsburgh. He is a co-founder and the Director of the Laboratory of Education and Research on Security Assured Information Systems (LERSAIS), which is NSA and DHS designated National Center of Academic Excellence in Information Assurance Education. He received his MS in Computer Science and $\mathrm{PhD}$ in Computer Engineering degrees from Purdue University in 1998 and 2003. His research interests include Security and Privacy of Distributed Systems, and Trust Management and Information Survivability. He is a recipient of the NSFCAREER award in 2006. His current research activities include advanced access control models, Security and Privacy issues in Cloud Computing and Collaborative systems, Social networks, and Intrusion detection/prevention systems. He served as Program Co-Chair for the IEEE IRI, the International Workshop on Information Assurance, and the International Workshop on Trusted Collaboration, IEEE/ICST CollaborateCom, COMPSAC2009 (as track chair) and ACM SACMAT2009. He has served as General chair of CollaborateCom (multiple times), SACMAT2010. He currently serves as a Steering committee member of CollaborateCom (co-chair) and SACMAT. He also served as an editorial board member of the International Journals of E-Business research, Network Security and Multimedia and Ubiquitous Engineering. He has served as a program committee member in many international conferences (over 70). He serves in the editorial review board of the International Journal of E-Business Research and the International Journal of Network Security. He is a co-editor of the book titled "Information Assurance: Dependability and Security of Networked Systems”.

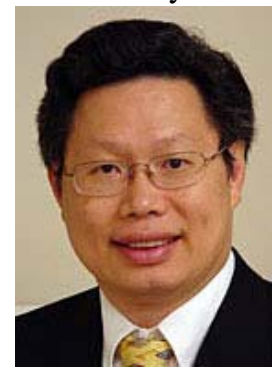

Calton Pu, (Georgia Institute of Technologies, USA) was born in Taiwan and grew up in Brazil. He received his $\mathrm{PhD}$ from University of Washington in 1986 and served on the faculty of Columbia University and Oregon Graduate Institute. Currently, he is holding the position of Professor and John P. Imlay, Jr. Chair in Software at the College of Computing, Georgia Institute of Technology. He is leading the Infosphere project, building software tools to support information flow-driven applications such as digital libraries and electronic commerce. Infosphere builds on his previous and ongoing research interests. First, he has been working on nextgeneration operating system kernels to achieve high performance, adaptiveness, security, and modularity, using program specialization, software feedback, and domainspecific languages. This area has included projects such as Synthetix, Immunix, Microlanguages, and Microfeedback, applied to distributed multimedia and system survivability. Second, he has been working on new data and transaction management by extending database technology. This area has included projects such as Epsilon Serializability, Reflective Transaction Framework, and Continual Queries over the Internet. His collaborations include applications of these techniques in scientific research on macromolecular structure data, weather data, and environmental data, as well as in industrial settings. He has published more than 70 journal papers and book chapters, 200 conference and refereed workshop papers. He served on more than 120 program committees, including the co-PC chairs of SRDS'95, ICDE'99, COOPIS'02, SRDS'03, DOA'07, DEBS'09, ICWS'10, CollaborateCom'11, ICAC'13, and cogeneral chair of ICDE'97, CIKM'01, ICDE'06, DEPSA'07, CEAS'07, SCC'08, CollaborateCom'08, World Service Congress'11, and CollaborateCom'12. 